\title{
Finite-Time State Estimation of Discrete-Time Linear Systems With Some Extensions. Application to Steering Lateral Vehicle Model
}

\author{
K. Chaib-Draa ${ }^{1}$, A. Zemouche ${ }^{2}$, R. Rajamani ${ }^{3}$, F. Bedouhene ${ }^{4}$, T.M. Laleg-Kirati ${ }^{5}$
}

\begin{abstract}
This paper presents novel exact finite-time estimation algorithms for linear discrete-time systems with extension to singular systems, under specific rank conditions. The proposed estimation algorithms are more general than the well-known deadbeat observers, which can provide finitetime estimation. Two new estimation schemes are proposed; the first scheme provides a direct and explicit estimation algorithm based on the use of delayed outputs, while the second scheme uses two combined asymptotic observers to recover in a finitetime the exact solution of the system. The effectiveness of the developed estimators is shown through application to a steering controlled lateral vehicle system where all states are estimated from look-ahead distance measurement.
\end{abstract}

\section{INTRODUCTION}

State estimation for any class of systems is a crucial task in control design. Indeed, estimation of the system state is an important step for designing control laws, fault diagnosis, or health monitoring [1], [2], [3], [4].

From asymptotic estimation point of view, if for nonlinear systems there is no general methodology, the problem is completely solved for linear systems. However, to achieve exact and finite-time estimation, even in linear case, the problem still remains open and there are some improvements. It is well known that the famous deadbeat observers solve this problem under specific constraints [5], [6], [7]. In this paper, we propose two alternative exact and finite-time estimation methodologies to solve the problem. The required assumptions are different from those required by deadbeat observers methodology based on nilpotency index of certain matrices.

The proposed two new finite-time estimation algorithms are summarized as follows:

- Direct and explicit estimation: This algorithm provides an explicit solution of the system in finite-time. This direct estimation approach is based on the use of delayed outputs to recover the solution of the system.

- Two observers-based estimation: This technique consists in combining two asymptotic state observers to

1 University of Luxembourg, Belval, Luxembourg (email: kh. chaibdraaegmail.com).

2 Université de Lorraine, CRAN CNRS UMR 7039, 54400 Cosnes et Romain, France (email: ali.zemoucheduniv-lorraine.fr).

${ }^{3}$ Laboratory for Innovations in Sensing, Estimation, and Control, Department of Mechanical Engineering, University of Minnesota, Minneapolis, USA. (email: rajamani@umn.edu).

4 Laboratoire de Mathématiques Pures et Appliquées, Université Mouloud Mammeri de Tizi-Ouzou, Algeria. (email: fbedouhene@ yahoo.fr).

${ }^{5}$ Department of Computer, Electrical and Mathematical Science andEngineering, KAUST, Thuwal, Saudi Arabia. (email: taousmeriem. laleg@kaust.edu.sa). reconstruct the solution of the system in finite-time. This second estimation algorithm is easier to implement numerically and may be used to investigate robustness to norm-bounded uncertainties or $\mathcal{L}_{2}$-bounded disturbances which are not considered in this preliminary version of the paper.

Extension to linear singular systems is provided under some rank conditions and after convenient matrix transformations. This extension is useful because it allows solving the estimation problem for linear systems with disturbances in the measurements and dynamics. It allows solving the issue of unknown input estimation, which is of considerable interest for control design schemes and diagnosis, since unknown inputs may represent actuator/sensor faults, uncertainties, or disturbances.

There are several issues to consider in the future to deepen the proposed methodology and to investigate performances and robustness of the proposed estimation schemes. Extensions to more general classes of systems are in progress, namely extension to switched linear systems, Linear Parameter Varying (LPV) systems, and specific nonlinear systems. Moreover, we aim to develop a systematic procedure for designing the estimation parameters.

The rest of this paper is organized as follows. Section II is divided into two subsections: Subsection II-A is devoted to the first estimation algorithm providing explicit solutions of the considered linear systems. The second estimation scheme is presented in Subsection II-B. An extension of the methodology to singular linear systems is provided in Section III. To show the validity and effectiveness of the proposed design algorithms, an application to steering controlled lateral vehicle system is presented in Section IV. Finally, Section V concludes this note.

\section{Preliminary Results on Exact Estimation For LINEAR Discrete-TIME SySTEMS}

\section{A. Explicit Solutions Using Delayed Outputs}

This section is devoted to a new exact finite-time estimation algorithm for linear systems described by the following equations:

$$
\left\{\begin{array}{l}
x_{k+1}=A x_{k}+B u_{k} \\
y_{k}=C x_{k}
\end{array}\right.
$$

where $x_{k}$ is the state of the system; $u_{k}$ is the control input, and $y_{k}$ is the vector of output measurements. Assume that the pair $(A, C)$ is observable. 
Let $L$ and $K$ be two matrices so that $(A-L C)$ and $(A-$ $K C)$ are Schur stable. Then, system (1) can be written as:

$$
\begin{gathered}
x_{k+1}=(A-L C) x_{k}+L y_{k}+B u_{k} \\
x_{k+1}=(A-K C) x_{k}+K y_{k}+B u_{k}
\end{gathered}
$$

By exploiting the above equations, we can provide an estimation of the state $x_{k}$ in finite-time. However, before stating the theorem and the exact finite-time estimation, we introduce the following lemma, which plays an important role in the existence of such an exact estimation in finite-time.

Lemma 1: Assume that the pair $(A, C)$ is observable. Then there exist $L, K$, and $m \geq 1$ such that the matrix

$$
\mathbb{E}_{m}=(A-L C)^{-m}-(A-K C)^{-m}
$$

is invertible.

Proof: Let us denote by $D(0 ; r)$ the disc centered at 0 with radius $r>0$. We denote by $D(0 ; r)$ its interior. For every $m \geq 1$, the matrix $\mathbb{E}_{m}$ is well-defined, since we can always choose both $L_{1}$ and $L_{2}$ such that all eigenvalues of $A-L_{i} C$ for all $i=1,2$ are located in $D(0 ; 1)-\{0\}$. With this choice, both $\left(A-L_{1} C\right)^{-m}$ and $\left(A-L_{2} C\right)^{-m}$ exist. Now, in view of the formula

$$
\mathbb{E}_{m}=\left(A-L_{1} C\right)^{-m}\left(I-\left(A-L_{1} C\right)^{m}\left(A-L_{2} C\right)^{-m}\right)
$$

and since $\left(A-L_{1} C\right)^{-m}$ is invertible for $m \geq 1$, we deduce that $\mathbb{E}_{m}$ is invertible if and only if $\mathbb{H}_{m}:=I-(A-$ $\left.L_{1} C\right)^{m}\left(A-L_{2} C\right)^{-m}$ is invertible. To get invertibility of $\mathbb{H}_{m}$, for every $m \geq 1$, it suffices to chose $L_{1}$ and $L_{2}$ such that

$$
\left\|\left(A-L_{1} C\right)^{m}\left(A-L_{2} C\right)^{-m}\right\|<1 .
$$

Observe that (5) holds for every $m \geq 1$ if

$$
\left\|\left(A-L_{1} C\right)\right\|\left\|\left(A-L_{2} C\right)^{-1}\right\|<1 .
$$

Indeed, this follows from the following inequalities:

$$
\begin{aligned}
\left\|\left(A-L_{1} C\right)^{m}\left(A-L_{2} C\right)^{-m}\right\| & \leq\left\|\left(A-L_{1} C\right)^{m}\right\|\left\|\left(A-L_{2} C\right)^{-m}\right\| \\
& \leq\left\|\left(A-L_{1} C\right)\right\|^{m}\left\|\left(A-L_{2} C\right)^{-1}\right\|^{m} .
\end{aligned}
$$

To get (5), it suffices then to place the eigenvalues of $A-$ $L_{1} C$ in $\stackrel{\circ}{D}\left(0 ; \frac{1}{2}\right)-\{0\}$, and the eigenvalues of $A-L_{2} C$ in $D(0 ; 1)-D\left(0 ; \frac{1}{2}\right)$ (or equivalently, the eigenvalues of $(A-$ $\left.L_{2} C\right)^{-1}$ in $D(0 ; 2)-\stackrel{\circ}{D}(0 ; 1)$. This allows us to get

$$
\begin{gathered}
\left.\left\|A-L_{1} C\right\| \in\right] 0, \frac{1}{2}[, \\
\left.\left.\left\|\left(A-L_{2} C\right)^{-1}\right\| \in\right] 1,2\right] .
\end{gathered}
$$

Inequality (5) is clearly achieved.

Remark 1: The proof of Lemma 1 provides a systematic method to design theoretically the gains $K$ and $L$ for any integer $m \geq 1$ so that the matrix $\mathbb{E}_{m}$ defined in (3) exists and invertible. This means that we can always find $K, L$ with $m=1$. However, this choice is not unique and for numerical considerations we can have $m>1$. Indeed, we can have the two following considerations:
- Schur stability is not necessary: The Schur stability of $A-L C$ and $A-K C$ is not necessary for the invertibility of $\mathbb{E}_{m}$. Indeed, it is possible to find matrices $L$ and $K$, with $A-L C$ and $A-K C$ not Schur stable (eigenvalues are not in $D(0 ; 1)$ ), and an integer $m \geq 1$ such that the matrix $\mathbb{E}_{m}$ is invertible. However, the observability of $(A, C)$ is necessary to systematically guarantee such a matrix $\mathbb{E}_{m}$.

- Condition number of $\mathbb{E}_{m}$ : Under the observability of the pair $(A, C)$, following the proof of Lemma 1, for any $m \geq 1$, there exist $L$ and $K$ such that $\mathbb{E}_{m}$ is invertible. However, for some choices of $L$ and $K$, the matrix $\mathbb{E}_{m}$ may be invertible but with high condition number, which leads to numerical troubles and then to bad estimation. For this reason, $m=1$ is not necessarily the best choice. A higher value of $m$ can be provided to improve the invertibility of $\mathbb{E}_{m}$ and to provide a better exact and finite-time estimation.

The explicit formula giving the exact estimation or the true value $x_{k}$ for any $k \geq m$ is presented in the following theorem.

Theorem 1: There exist $L, K$ and $m \geq 1$ so that the solution of (1) satisfies $\forall k \geq m$ :

$$
\begin{aligned}
x_{k}=\mathbb{E}_{m}^{-1} \sum_{j=1}^{m}[ & (A-L C)^{j-m-1} L \\
& \left.-(A-K C)^{j-m-1} K\right] y_{k-j} \\
+\mathbb{E}_{m}^{-1} \sum_{j=1}^{m}\left[(A-L C)^{j-m-1}\right. & {\left[(A-K C)^{j-m-1}\right] B u_{k-j} }
\end{aligned}
$$

Proof: We can show easily, after some iterations, that for $k \geq m$, we have

$$
\begin{aligned}
x_{k}=(A-L C)^{m} x_{k-m} & +\sum_{j=1}^{m}(A-L C)^{j-1} L y_{k-j} \\
& +\sum_{j=1}^{m}(A-L C)^{j-1} B u_{k-j} \\
x_{k}=(A-K C)^{m} x_{k-m}+ & \sum_{j=1}^{m}(A-K C)^{j-1} K y_{k-j} \\
& +\sum_{j=1}^{m}(A-K C)^{j-1} B u_{k-j}
\end{aligned}
$$

By multiplying equations (7a) and (7b) by $(A-L C)^{-m}$ and $(A-K C)^{-m}$, respectively, we get

$$
\begin{aligned}
(A-L C)^{-m} x_{k}=x_{k-m} & +\sum_{j=1}^{m}(A-L C)^{j-m-1} L y_{k-j} \\
& +\sum_{j=1}^{m}(A-L C)^{j-m-1} B u_{k-j}
\end{aligned}
$$




$$
\begin{aligned}
(A-K C)^{-m} x_{k}=x_{k-m} & +\sum_{j=1}^{m}(A-K C)^{j-m-1} K y_{k-j} \\
& +\sum_{j=1}^{m}(A-K C)^{j-m-1} B u_{k-j}
\end{aligned}
$$

By subtracting (8b) from (8a), it follows that

$$
\begin{aligned}
\mathbb{E}_{m} x_{k}= & \sum_{j=1}^{m}\left[(A-L C)^{j-m-1} L\right. \\
& \left.-(A-K C)^{j-m-1} K\right] y_{k-j} \\
+ & \sum_{j=1}^{m}\left[(A-L C)^{j-m-1}\right. \\
& \left.-(A-K C)^{j-m-1}\right] B u_{k-j}
\end{aligned}
$$

Finally, since from Lemma 1 , there exists $m \geq 1$ so that $\mathbb{E}_{m}$ is invertible, then we can conclude.

\section{B. Finite-Time Estimation Using Two Combined Observers}

Unlike the previous section where we used a sum of delayed outputs weighted by powers of $A-L C$ and $A-K C$, this section is devoted to estimate the solutions of the considered system by using two different asymptotic state observers. By using tools borrowed from the continuoustime results in [8] and [9], we get an exact estimation of the solution without using explicitly the delayed outputs. Indeed, the delayed output measurements are hidden and appear implicitly in the states of the intermediate observers. This way to provide an exact estimation of the state $x_{k}$ is more suitable for practical implementation point of view.

Consider the following two state observers corresponding to (1) and described by the equations:

$$
\left\{\begin{array}{l}
\zeta_{k+1}=A \zeta_{k}+B u_{k}+L\left(y_{k}-C \zeta_{k}\right) \\
\zeta_{0} \in \mathbb{R}^{n}
\end{array}\right.
$$

and

$$
\left\{\begin{array}{l}
\eta_{k+1}=A \eta_{k}+B u_{k}+K\left(y_{k}-C \eta_{k}\right) \\
\eta_{0} \in \mathbb{R}^{n}
\end{array}\right.
$$

where $L$ a nd $K$ are the two observer gains to be determined.

Theorem 2: Assume that there exist $L, K$, and $m \geq 1$ so that the matrix $\mathbb{E}_{m}$ exists and invertible. Then, the solution of system (1) satisfies $\forall k \geq m$ :

$$
\begin{aligned}
x_{k}=\mathbb{E}_{m}^{-1} & {\left[(A-L C)^{-m} \zeta_{k}-\zeta_{k-m}\right.} \\
& \left.-(A-K C)^{-m} \eta_{k}+\eta_{k-m}\right] .
\end{aligned}
$$

Proof: By reasoning as in the previous section, we can easily write $\zeta_{k}$ and $\eta_{k}$ as follows:

$$
\begin{aligned}
\zeta_{k}= & (A-L C)^{m} \zeta_{k-m}+\sum_{j=1}^{m}(A-L C)^{j-1} L y_{k-j} \\
& +\sum_{j=1}^{m}(A-L C)^{j-1} B u_{k-j} \\
\eta_{k}= & (A-K C)^{m} \eta_{k-m}+\sum_{j=1}^{m}(A-K C)^{j-1} K y_{k-j} \\
& +\sum_{j=1}^{m}(A-K C)^{j-1} B u_{k-j} .
\end{aligned}
$$

It follows that

$$
\begin{aligned}
\Sigma_{m}= & (A-L C)^{-m} \zeta_{k}-\zeta_{k-m} \\
& -(A-K C)^{-m} \eta_{k}+\eta_{k-m}
\end{aligned}
$$

where

$$
\begin{aligned}
\Sigma_{m} \triangleq \sum_{j=1}^{m}\left[(A-L C)^{j-m-1} L\right. \\
\left.-(A-K C)^{j-m-1} K\right] y_{k-j} \\
+\sum_{j=1}^{m}\left[(A-L C)^{j-m-1}\right. \\
\left.-(A-K C)^{j-m-1}\right] B u_{k-j} .
\end{aligned}
$$

Then by substituting (15) in (6), the relation (12) is inferred.

\section{EXACT Finite-Time ESTIMATION FOR LiNEAR SINGULAR SYSTEMS}

In this section, we propose an extension of the proposed methodology to a class of descriptor systems for which there are no available results in the literature on finite-time estimation.

\section{A. System description}

Consider the descriptor systems described by the following equations:

$$
\left\{\begin{array}{l}
E_{\zeta} \zeta_{k+1}=A_{\zeta} \zeta_{k}+B_{\zeta} v_{k} \\
y_{k}=C_{\zeta} \zeta_{k}
\end{array}\right.
$$

where $E_{\zeta}, A_{\zeta}, B_{\zeta}$ and $C_{\zeta}$ are constant matrices of appropriate dimensions. The state vector is $\zeta_{k} \in \mathbb{R}^{n_{\zeta}}$ and the control input vector is $v_{k} \in \mathbb{R}^{m_{\zeta}}$. Let us introduce the following rank condition, which is standard in descriptor systems theory.

Assumption 1: The matrices $E_{\zeta}$ and $C_{\zeta}$ satisfy the following condition:

$$
\operatorname{rank}\left(\left[\begin{array}{l}
E_{\zeta} \\
C_{\zeta}
\end{array}\right]\right)=n_{\zeta}
$$

Condition (18) is necessary to guarantee existence of an observer for (17). 


\section{B. System transformation and estimation}

System (17) can be rewritten under the form

$$
\left[\begin{array}{c}
E_{\zeta} \\
C_{\zeta}
\end{array}\right] \zeta_{k+1}=\left[\begin{array}{c}
A_{\zeta} \\
0
\end{array}\right] \zeta_{k}+\left[\begin{array}{c}
0 \\
\mathbb{I}_{p}
\end{array}\right] y_{k+1}+\left[\begin{array}{c}
B_{\zeta} \\
0
\end{array}\right] v_{k}
$$

From Assumption 1, the matrix

$$
\left[\begin{array}{l}
E_{\zeta} \\
C_{\zeta}
\end{array}\right]^{\top}\left[\begin{array}{l}
E_{\zeta} \\
C_{\zeta}
\end{array}\right]
$$

is invertible. It follows that after pre-multiplying (19) by $\left[\begin{array}{l}E_{\zeta} \\ C_{\zeta}\end{array}\right]^{\top}$, and using the notation

$$
\left[\begin{array}{ll}
P_{\zeta} & Q_{\zeta}
\end{array}\right] \triangleq\left(\left[\begin{array}{l}
E_{\zeta} \\
C_{\zeta}
\end{array}\right]^{\top}\left[\begin{array}{l}
E_{\zeta} \\
C_{\zeta}
\end{array}\right]\right)^{-1}\left[\begin{array}{l}
E_{\zeta} \\
C_{\zeta}
\end{array}\right]^{\top}
$$

we get the new structure of (19):

$$
\zeta_{k+1}=P_{\zeta} A_{\zeta} \zeta_{k}+\left[\begin{array}{ll}
P_{\zeta} B_{\zeta} & Q_{\zeta}
\end{array}\right]\left[\begin{array}{c}
v_{k} \\
y_{k+1}
\end{array}\right]
$$

which is exactly under the form (1) with

$$
A \triangleq P_{\zeta} A_{\zeta}, \quad B \triangleq\left[P_{\zeta} B_{\zeta} \quad Q_{\zeta}\right]
$$

and

$$
u_{k} \triangleq\left[\begin{array}{c}
v_{k} \\
y_{k+1}
\end{array}\right]
$$

Hence, we can apply directly the results of Section II to get exact finite-time estimation of $\zeta$.

1) Explicit solution: By analogy to Theorem 1, if there exist $L, K$, and $m \geq 1$ so that the matrix $\mathbb{E}_{m}$, defined by

$$
\mathbb{E}_{m}=\left(P_{\zeta} A_{\zeta}-L C\right)^{-m}-\left(P_{\zeta} A_{\zeta}-K C\right)^{-m}
$$

is invertible, then the solution of (17) satisfies $\forall k \geq m$ :

$$
\begin{aligned}
& \zeta_{k}=\mathbb{E}_{m}^{-1} \sum_{j=1}^{m} {\left[\left(P_{\zeta} A_{\zeta}-L C\right)^{j-m-1} L\right.} \\
&\left.-\left(P_{\zeta} A_{\zeta}-K C\right)^{j-m-1} K\right] y_{k-j} \\
&+\mathbb{E}_{m}^{-1} \sum_{j=1}^{m}\left[\left(P_{\zeta} A_{\zeta}-L C\right)^{j-m-1}\right.\left.-\left(P_{\zeta} A_{\zeta}-K C\right)^{j-m-1}\right] \times \\
& {\left[\begin{array}{ll}
P_{\zeta} B_{\zeta} & Q_{\zeta}
\end{array}\right]\left[\begin{array}{c}
v_{k-j} \\
y_{k-j+1}
\end{array}\right] . }
\end{aligned}
$$

2) Two-observers based estimation: By analogy to Theorem 2 , if there exist $L, K$, and $m \geq 1$ so that the matrix
$\mathbb{E}_{m}$ defined by (21) is invertible, then $\forall k \geq m$ :

$$
\begin{gathered}
\zeta_{k}=\mathbb{E}_{m}^{-1}\left[\left(P_{\zeta} A_{\zeta}-L C\right)^{-m} \xi_{k}-\xi_{k-m}\right. \\
\left.-\left(P_{\zeta} A_{\zeta}-K C\right)^{-m} \eta_{k}+\eta_{k-m}\right] ; \\
\xi_{k+1}=P_{\zeta} A_{\zeta} \xi_{k}+\left[\begin{array}{ll}
P_{\zeta} B_{\zeta} & Q_{\zeta}
\end{array}\right]\left[\begin{array}{c}
v_{k} \\
y_{k+1}
\end{array}\right]+L\left(y_{k}-C \xi_{k}\right) \\
\eta_{k+1}=P_{\zeta} A_{\zeta} \eta_{k}+\left[\begin{array}{ll}
P_{\zeta} B_{\zeta} & Q_{\zeta}
\end{array}\right]\left[\begin{array}{c}
v_{k} \\
y_{k+1}
\end{array}\right]+K\left(y_{k}-C \eta_{k}\right) .
\end{gathered}
$$

\section{Application to linear lateral Vehicle model}

We will illustrate the proposed algorithms on a linear system application of a steering-controlled lateral vehicle model. Through this model, we will validate the design algorithm (6) and give comparisons to asymptotic estimation.

\section{A. Model description}

The linear lateral vehicle model is described under the form (1) after Euler discretization with sampling period $T_{e}=$ 0.01 . The system matrices in the continuous-time are given as follows [10]:

$$
A=\left[\begin{array}{cccc}
0 & 1 & 0 & 0 \\
0 & -\frac{2\left(C_{f}+C_{r}\right)}{M V_{x}} & \frac{2\left(C_{f}+C_{r}\right)}{M} & -\frac{2\left(C_{f} l_{f}+C_{r} l_{r}\right)}{M V_{x}} \\
0 & 0 & 0 & 1 \\
0 & -\frac{2\left(l_{f} C_{f}-l_{r} C_{r}\right)}{I_{z} V_{x}} & \frac{2\left(l_{f} C_{f}-l_{r} C_{r}\right)}{I_{z}} & -\frac{2\left(l_{f}{ }^{2} C_{f}+l_{r}{ }^{2} C_{r}\right)}{I_{z} V_{x}}
\end{array}\right]
$$

$$
B=\left[\begin{array}{c}
0 \\
\frac{2 C_{f}}{M} \\
0 \\
\frac{2 l_{f} C_{f}}{I_{z}}
\end{array}\right], \quad C=\left[\begin{array}{llll}
1 & 0 & d_{s} & 0
\end{array}\right] .
$$

The state vector $x \in \mathbb{R}^{4}$ and the control input consists of the steering angle $u$. The parameters $M, I_{z}, V_{x}, l_{f}$ and $l_{r}$ are the mass, yaw inertia, longitudinal velocity, the length of front end and rear end to the center of gravity of the vehicle respectively. For more details on the lateral model, we refer the reader to [10]. It should be noticed that the system is unstable (Steering control always has an unstable plant model). In order to run the numerical simulation, we take $M=1573, I_{z}=2873, l_{f}=1.1, l_{r}=1.58, C_{f}=$ $80000, C_{r}=80000, d_{s}=2$, and we initialize the model system by $x_{0}=\left[\begin{array}{llll}10 & 0 & 3 & -5\end{array}\right]^{T}$ and the estimated state by $\hat{x}_{0}=\left[\begin{array}{llll}100 & 100 & 100 & 100\end{array}\right]^{T}$.

\section{B. Exact finite-time estimation}

By using pole assignment with eigenvalues $\{0.5,0.1,0.3,0.2\}$ and $\{0.02,0.01,0.07,0.03\}$ for $A-L C$ and $A-K C$, respectively, we found

$$
L=\left[\begin{array}{c}
0.5316 \\
2.0824 \\
0.1596 \\
-0.6307
\end{array}\right], \quad K=\left[\begin{array}{l}
1.1432 \\
5.6539 \\
0.3388 \\
0.1713
\end{array}\right],
$$


which guarantee existence and invertibility of the matrix $\mathbb{E}_{4}$. Even if the explicit estimated state is badly initialized, it is quite clear from Figures 1-4 that the estimation is achieved on finite-time after $m=4$. The input used for simulation is a sinusoidal signal $u=2 \sin \left(\frac{2 \pi}{15} t\right)$. Compared to the asymptotic observer, the proposed estimation in (6) converges quickly.

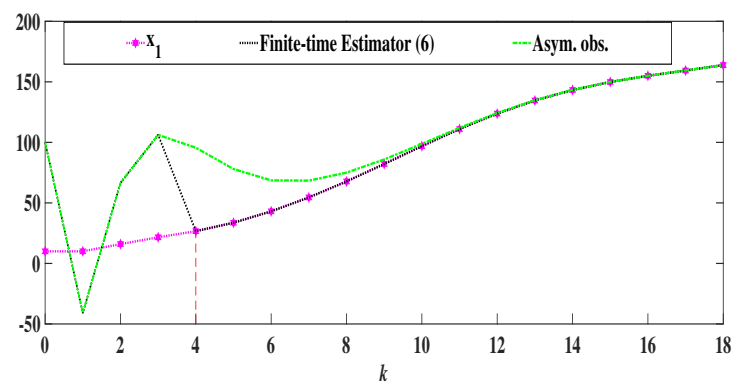

Fig. 1. $x_{1}$ and its estimations.

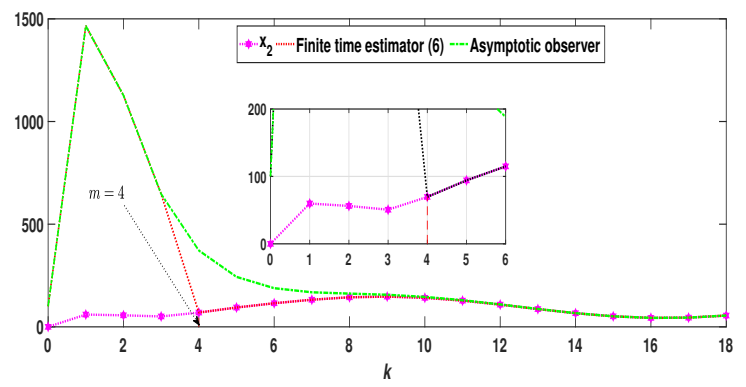

Fig. 2. $x_{2}$ and its estimations.

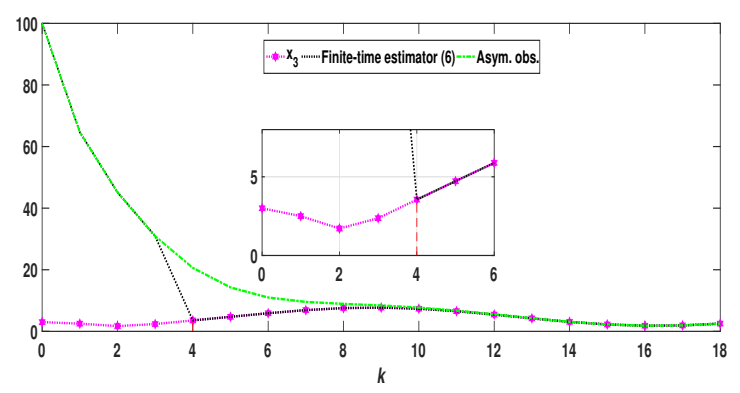

Fig. 3. $x_{3}$ and its estimations.

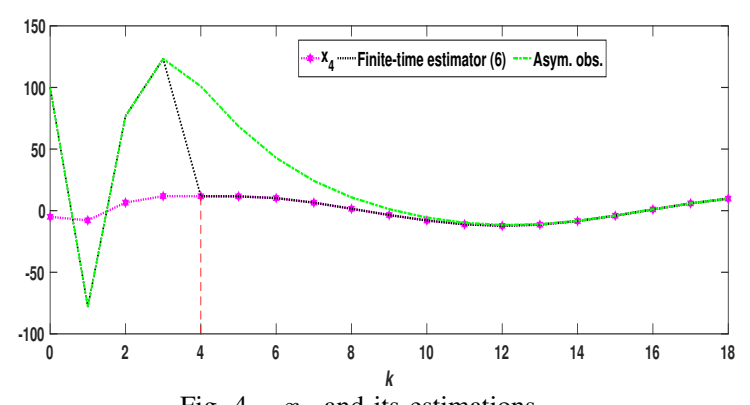

Fig. 4. $x_{4}$ and its estimations.

\section{CONCLUSION}

Under invertibility conditions of certain matrices, we provided two algorithms for exact finite time estimation of linear discrete-time systems. Under a certain rank condition and after some matrix transformation of the system, an extension to a class of linear singular systems is proposed. A linear steering lateral vehicle model served as a numerical example to demonstrate effectiveness of the proposed exact finite-time estimation algorithms. Due to the satisfactory results, we target in the near future to investigate the following issues:

- Design of the estimation parameters: All the issues related to the design of the estimation parameters, namely the observer gains and the finite-time are not considered in this paper in a deepen way. Only few indications are provided.

- Robustness and performance issues: The proposed estimation schemes will be generalized to systems in the presence of uncertainties. Then, we will develop new approximate estimation algorithms.

- Extension to nonlinear systems, LPV systems, Switched systems: One of the main objective consists in generalizing the methodology to a class of nonlinear systems that can be transformed into a linear form. An extension to Linear Parameter Varying (LPV) systems is an alternative solution to tackle nonlinear systems.

- Link to deadbeat observers and modulating functions based estimation: Establishing comparisons of the proposed methodology with the classical deadbeat observers [6] and the modulating functions based algorithms [11] is a challenge we aim to achieve.

\section{REFERENCES}

[1] Z. Gao and D. W. C. Ho, "State/noise estimator for descriptor systems with application to sensor fault diagnosis," IEEE Transactions on Signal Processing, vol. 54, no. 4, pp. 1316-1326, 2006.

[2] E. Alcorta-Garcia and P. M. Frank, "Deterministic nonlinear observerbased approaches to fault diagnosis: a survey," Control Engineering Practice, vol. 5, no. 5, pp. 663-670, 1997.

[3] R. Marino and P. Tomei, Nonlinear Control Design. Prentice Hall, 1995.

[4] M. Arcak and P. Kokotovic, "Observer-based control of systems with slope-restricted nonlinearities," IEEE Transactions on Automatic Control, vol. 46, no. 7, pp. 1146-1150, 2001.

[5] S. E. Tuna, "Deadbeat observer: Construction via sets," in 2011 American Control Conference, ACC 2011, San Francisco, CA, USA, July 2011.

[6] M. E. Valcher and J. C. Willems, "Dead beat observer synthesis," Systems \& Control Letters, vol. 37, no. 5, pp. 285 - 292, 1999.

[7] S. Kim and P. Park, " $\mathcal{H}_{\infty}$ output-feedback control based on an fir-type quasi-deadbeat observer," IEEE Transactions on Automatic Control, vol. 53, pp. 1492-1498, 2008.

[8] R. Engel and G. Kreisselmeier, "A continuous-time observer which converges in finite time," IEEE Transactions on Automatic Control, vol. 47, no. 7, pp. 1202-1204, Jul 2002.

[9] F. Mazenc, E. Fridman, and W. Djema, "Estimation of solutions of observable nonlinear systems with disturbances," Systems \& Control Letters, vol. 79, pp. 47 - 58, 2015.

[10] R. Rajamani, Vehicle Dynamics and Control. 2nd edition, Springer Verlag, 2012.

[11] D.-Y. Liu, T.-M. Laleg-Kirati, W. Perruquetti, and O. Gibaru, "Nonasymptotic state estimation for a class of linear time-varying systems with unknown inputs," IFAC Proceedings Volumes, vol. 47, no. 3, pp. 3732-3738, 2014. 